\title{
The Seasons in the Music Lesson - A Resourceful Topic for the Acquisition of Musical Means of Expression and the Development of Students' Creative Thinking
}

Krassimira Georgieva Fileva-Ruseva Ph.D ${ }^{1 *}$, Asen Diamandiev ${ }^{2}$

${ }^{1}$ Associate Professor, ${ }^{2}$ Professor, Academy of Music, Dance and Fine Arts "" - Plovdiv, Bulgaria, European Union

DOI: $10.36348 /$ sjhss.2020.v05i10.008

| Received: 24.09.2020 | Accepted: 09.10.2020 | Published: 11.10.2020

*Corresponding author: Krassimira Georgieva Fileva-Ruseva

\section{Abstract}

The educational set for the music lesson in Bulgarian school contain a printed edition of the textbook with the basic information intended for acquisition; a teacher's book which contains additional information on each topic that will be taught and a suggested plan for each lesson; a student's book with supplementary questions and tasks, suitable for both classroom work and homework; a set of CDs with the music for listening and the songs for singing; an electronic textbook, combining the content of the printed edition of the textbook, all musical works for listening and songs for performing, offered with soloists included, and - in a separate file - only the instrumental accompaniment suitable for performing simbeck, as well as many additional questions tasks, project ideas, a variety of illustrative material - videos, additional musical works, diagrams, tables, photos, poems. The availability of the electronic textbook from any device with internet makes it especially up to date in the conditions of distance learning imposed by the pandemic. In the present study, the tasks dedicated to the topic of the seasons from the printed and electronic textbooks of Prosveta publishing house [1-3] are subjected to theoretical analysis. The results of a pedagogical experiment confirmed those of the theoretical analysis, proving significantly higher success rate, higher potential for unconventional thinking and increased interest in music lessons in students of the $2 \mathrm{nd}$, 3rd and 4th grade of general education school after the experimental education.

Keywords: seasons, musical means of expression, imagination, creative thinking, music textbook, music lesson, general education school, electronic textbook, student.

Copyright (C) 2020 The Author(s): This is an open-access article distributed under the terms of the Creative Commons Attribution 4.0 International License (CC BY-NC 4.0) which permits unrestricted use, distribution, and reproduction in any medium for non-commercial use provided the original author and source are credited.

\section{INTRODUCTION}

The educational sets for the general education school in Bulgaria contain:

- $\quad$ Printed edition of the textbook, including the richly illustrated basic information that the students should learn;

- A teacher's book, in which the pedagogue can find the additional information which to present to the students, as well as suggested plans for each lesson;

- A student's book, including additional tasks for each lesson;

- A set of CDs with recordings of the works to be listened to and the songs to be performed by the students;

- An electronic textbook.
The electronic textbook is an electronic version of the printed edition of the textbook, including also

The entire content of the CDs of the educational set, i.e. all musical works determined for listening by the students and all songs provided for learning and performing, furthermore, for each song a file with a recording of the performance including the solo part of the song has been created to be used as a reference and a separate file with a recording of only the accompaniment (instrumental) that students can use for their performance simbeck.

Many additional tasks and questions to support the comprehension of the taught educational information and to serve to verify the learned;

Additional illustrative material - musical works, videos, photos, some of which - shown individually, others, according to the objectives of the 
lesson - arranged in photo galleries, poems, diagrams, tables.

The electronic textbook is accessible from any electronic device with internet connection (computer, phone, tablet), which makes it extremely "mobile" and convenient to use both in class and at home. The remote conduct of some classes and mixed (face-to-face and distance) lessons imposed by the new conditions makes electronic textbooks a particularly actual and indispensable assistant to both the teacher and the students.

The four seasons we enjoy in the countries with temperate climate - the fresh and tender diversity and caress of spring with the magical changing aromas of flowering plants, the bright, sunny colors of the summer, which students also associate with the carefreeness of the long summer vacation and the adventures of the summer games, the lavish multicolor of blazing, warm and earthy colors of autumn, which generously gives us fruits and the purity of the black and white winter, with the exquisite crystals that it draws on the windows, with the frost garlands that it wraps around the branches of the trees and bushes, with the bizarre draperies of icicles that it hangs from the roofs of the buildings - create bright and lasting impressions in everyone who sees them. In this sense, the seasons:

They provide many opportunities for the development of children's imagination and creative thinking. Appropriately used by the pedagogue, vivid visual impressions can be recreated in a beautiful and exciting way;

They are a particularly favorable topic for presenting to the students of the musical means of expression, united in the work by the idea of sound imaging, i.e. aimed at presenting the emotional and color characteristics of the respective season, and in some cases - to sound imitation of seasonal natural phenomena and human activities. In this way, the disclosure of the infinite sound-imaging and soundimitating possibilities of music becomes vivid, intuitive-immediate, fascinating and directly influencing the children's imagination, which, under the impact of music, completes its own natural pictures.

The tasks created in the electronic textbooks on the topics for the four seasons are closely related to the study material provided for listening and performing.

Scope of the study. The present study is focused on the topic of the four seasons from the electronic textbooks for the 2nd, 3rd and 4th grade of the Bulgarian general education school of P. Mincheva and author's team [1-3]. The educational set of the same authors for the 1st grade is not discussed in the present study, because in it the tasks related to the seasons are still too few for a targeted pedagogical impact to be observed. The curriculum of 5th, 6th, 7th, 8th and 9th grade students, who also study music, is occupied with much information, so in order to free up time for teaching it, the topic of the seasons has not been developed consistently. For this reason, textbooks for higher-grade students also are not the subject of the study. I focus on the educational sets of an author's team led by Professor Penka Mincheva, because, on the one hand, the theme of the seasons is developed consistently, with many creative tasks, and on the other hand the electronic textbooks include a very large number and diverse additional resources. In the educational sets of the other authors teams, despite its value for presenting the musical means of expression, for integrative connections with other educational disciplines and for solving creative tasks, the theme for the seasons is not purposefully developed, usually not developed at all, in some of the sets only several songs are included, and the electronic textbooks contain a few and unvaried additional resources.

The topic of the seasons attracted my attention because of the variety of opportunities it offers for the development of imagination and creative thinking of the adolescents - qualities that are especially necessary for a contemporary person. The teaching of the musical means of expression, i.e. the students learning "to speak" the language of music is also achievable and favored by the theme of the seasons.

Many other electronic resources have been developed and proposed for use in the electronic textbooks discussed in this article, but they relate to topics other than the one under consideration, so they are not the subject of analysis in this study.

The aim of the present study is to prove the effectiveness of the tasks from the above-mentioned educational sets for the learning of the musical means of expression and the development of the students' creative thinking.

Taking into account the availability of the electronic textbook, it becomes especially useful and necessary in a pandemic, where it is often necessary the form of distance learning to be used. For this reason, the analysis of its content is especially relevant.

The need for a creative approach to problems is also undoubtedly a topical issue. Exploring the possibilities for the development of creative thinking in school education and especially in the creation of textbooks for this education, as well as proposing new approaches, can help create better textbooks, and hence - increase the creative potential of the adolescents. 
The topicality of the proposed study can also be confirmed by referring to studies conducted by other scientists.

In her research, the prominent Bulgarian music theorist, solfeggio and music pedagogue Professor Penka Mincheva developed three main types of ideas that have become the guideline for the development of the Bulgarian mass music education:

The idea of implementation the mass music education on the basis of the native music folklore [4] knowledge of folklore music of one's own country is the musical equivalent of speaking one's mother language;

The idea of the definitely positive influence of the purposeful music lessons on the development of the learner's intellect [5]. It is mainly based on:

The way of recording the musical thought - the notation - is based on the note sign, which contains two different types of information - for pitch and for duration of the tone;

Receiving the sound music information - the perception of music - through hearing. As the auditory analyzer is not the most commonly used in everyday human practice, its purposeful and varied use in the musical activities carried out in the music lesson in the general education school is beneficial for the general intellectual development of the adolescents.

The idea of using works of other arts $[6,7]$ in order to deepen and enrich the impressions of the perceived music in the education in the general education school, as well as, in the long term - to develop the emotional intelligence of students. Mincheva [6] expresses the following opinion: "A productive form of work to stimulate the figurative thinking of the students is to make a connection between the arts in the music lesson. In fact, this is one of the most promising forms of using the integrative approach in education not only between different arts, but also between different subjects, such as history, religion, philosophy and etc. Viewing reproductions of paintings by great artists, listening to poems and so on should be used not only when there are external, insignificant similarities between the works, such as the same title, but also when the connection refers to the internal content - an analogous emotional state is recreated, in which each work of the individual arts complements, enriches, stimulates the experience and awareness of the other works, object of perception and comparison. Thus, the impressions of different arts complement each other, which significantly increase the effect of the emotional response.

A similar result is obtained when students are asked to convey through their drawing or verse the mood of a musical work they have listened to"[6].
To some extent, the present study continues and complements the results achieved by P. Mincheva. Among the benefits of the activity of drawing on the motor and intellectual development of the child, which the artist and children's drawing teacher Daniela Seferinkina [8] brings out, are: improving the fine motor skills; refining the coordination of both hands, as these two elements have a beneficial effect on the brainmotor apparatus relationship; improving observation, concentration and self-discipline; achieving emotional stability; developing imagination and creativity. The author states the following: "Drawing develops unconventional thinking and allows children to develop their imagination and recreate it on a white sheet. When encouraged to create something new, kids develop a real and intense sense of creation that will be very important in their lives. It will give them the opportunity to be bold and creative in solving problems, to find new ways and to improve existing ways of thinking and acting" [8].

The use of works of other arts is one of the forms of work that is relied on for developing the creative potential of the adolescents in the lessons dedicated to the seasons in the educational sets considered in this study, and the ability of unconventional thinking is an element of varied kinds of intelligence.

I. Shindarov [9] expresses the opinion that "When during drawing, children focus on the process, they acquire mental habits - comparing objects, analyzing, generalizing" [9].

The music pedagogue Professor Sevdalina Dimitrova [10] in an extensive study compares the electronic textbooks for the $1 \mathrm{st}$ - 4th grade of general education school from three educational sets, using 22 different criteria for comparison, among which of special importance is the presence of tasks related to the development of logical thinking and presentation of a theoretical-cognitive text. With this, Dimitrova indirectly confirms the importance of electronic resources for the development of students' intelligence.

According to Asya Veleva, "the elemental development of creative powers and abilities cannot be relied on. Even at preschool and general education school age, educational tasks to stimulate creativity should be introduced and adequate procedures should be developed"[11]. The researcher believes that the creative product should be defined as such by the following criteria - "criteria for defining a product as creative include: significance (individual or social), novelty (transformation, new construction, new connections between ideas, aesthetic qualities) and meaningfulness"[11]. The author offers ideas for the development of creative abilities, as well as several types of tasks for assessing the creative potential of the children. What the approaches to evaluation have in 
common is that the highest evaluation is given to the idea to ignore the usual (usual appearance, purpose, combinations with other objects), to look for the nonstandard, to change the object itself, to leave a situation or meaning with which as a rule, a certain object is connected by replacing the situation with unreal one or giving the object an unusual meaning, statistically rare children's suggestions and the ability to explain the chosen solution.

\section{EXPERIMENTAL SECTION MATERIALS AND METHODS}

2.1.1. Materials, i.e. the object of research are the electronic and printed textbooks for the 2nd, 3rd and 4th grade of the general education school in Bulgaria of an author's team headed by Professor Penka Mincheva [1-3]. The present study also includes students from the 2nd, 3rd and 4th grade of the general education school in Bulgaria, studying on the cited educational sets and forming experimental groups, as well as students of the same age using educational sets of another author's team. The latter form the control groups in a pedagogical experiment.

2.1.2. The methods used in the study are:

2.1.2.1. Theoretical analysis of the tasks related to the seasons in the three cited above printed and electronic textbooks;

2.1.2.2. Analysis of the literature on the issues addressed in the study;

2.1.2.3. Synthesis between the arts;

2.1.2.4. Integrative connections with the subject's literature, fine arts;

2.1.2.5. Formal analysis and analysis of the emotional content of a music work;

2.1.2.6. Analysis of the emotional content of a poetic text;

2.1.2.7. Experimental verification of the results of the theoretical study;

2.1.2.8. Diagnostic test for creativity [11].

\section{Ethical considerations}

The experiment, conducted for practical verification of the results of the theoretical analyzes, in fact is teaching students in a general education school on educational sets of two author teams. A total of six educational sets used in the experiment, have been approved for issuance and implementation in practice by the Ministry of Education and Science of the Republic of Bulgaria, as well as, according to the laws of our country as a second stage of the verification by 1800 teachers from Bulgarian general education schools. The educational sets on which the experiment was conducted, from the moment of their issuance, i.e. from 2017 - publication of the textbooks for the 2nd grade, from 2018 - the year of publication of the textbooks for 3rd grade and from 2019 - issuance of the textbooks for the 4 th grade, are widely used in general education schools in Bulgaria. This entire means that additional approval of the experiment by an ethics committee is not necessary.

The diagnostic test for creative abilities was conducted in a study by A. Veleva [11] and published. I use it when correctly taking into account the author's contribution of the researcher of children's creativity.

\section{Theoretical analysis of electronic resources, object of the study}

The autumn is associated with the farewell to the hot sunbeams and the carefreeness of the summer, with the songbirds that fly south, but also with the coloring of the leaves of plants in an incredible variety of shades of all the warm colors, decorating and coloring with their warmth our daily lives too.

In the electronic textbook for the 2nd grade [1] the first task related to a season is how the children will draw the autumn. To facilitate the task, five answers are proposed, with the possibility to choose more than one answer. The first, second and fifth listed answers - with cheerful sun; with snow-covered houses and streets; and with blooming flowers illustrate other seasons. The correct answers - those that are related to autumn natural phenomena - are the third - with leaves falling from the trees; and the fourth - with birds flying in flocks. Some resourcefulness is needed here to choose the correct answers. Since children prefer summer and winter - the seasons in which they have vacations, and the conditions allow for the most exciting games to take place, respectively - these are the seasons that leave the brightest and longest lasting memories, so the emotional reaction is focused first on the answers, characterizing summer or winter. In order to choose the correct answers, it is necessary to ignore the preference based on emotions, to evaluate all the answers and to choose the appropriate ones. However, in general, the task does not complicate the second grade children. This task is preparatory to the next, which is a little more complicated.

In the next electronic resource it is required to listen to the piano piece by Vasil Kazandjiev "Autumn rain knocks on the window" and to determine what autumn phenomena the famous Bulgarian composer has depicted with music. Among the proposed answers are those related to visual impressions, such as "an icy road" and "a blooming snowdrop", sound phenomena such as "a bird tweeting", sound-sensational, such as "cold and stormy wind" as well as visual but emotionally colored descriptions, such as the correct answer "with rain and sadness." The first three answers listed - "an icy road", "a blooming snowdrop" and "a bird tweeting" - are typical of other seasons, so they quickly are rejected as possible answers. The wind is not a rare phenomenon during the season, but it is completely absent from Kazandjiev's musical picture. On the other hand, in the play there are only two indications of dynamics - piano - set at the beginning 
and diminuendo - in the last four bars. This means that the composer requires a predominantly dynamically monotonous performance. The often repeated rhythmic motif consisting of a quarter note and two eighths (example № 1) creates an association with the tapping of raindrops on the window, and the accompanying voice almost all the time has the rhythmic structure of a quarter pause and a retained quarter note.

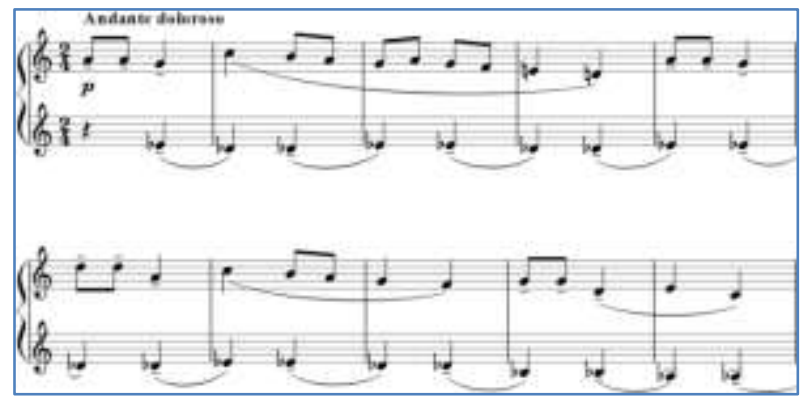

Example-1: Vasil Kazandjiev - "Autumn rain knocks on the window" bars 1 - 10

A certain exception to this is the conclusion of the piece, where a canon is composed on the rhythmic motif of a quarter note and two eighths, but there is also an indication of retention for the quarter, which creates continuity with the previous part (example № 2).

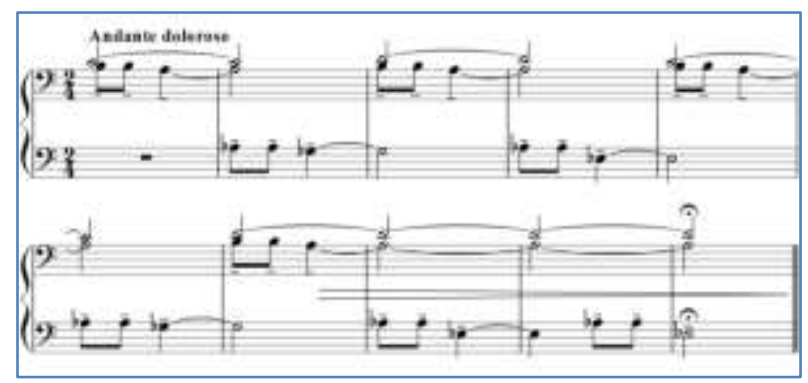

Example-2: Vasil Kazandjiev - "autumn rain knocks on the window" bars 17 - 26

This combination of the means of expression unequivocally points to the correct answer - "with rain and sadness". It reflects the deliberately sought by various musical means monotony of sound which is a peculiar and very successful tonal portrait of the despondency of late autumn, when the multicolor of the leaves has passed, replaced by the bare branches of the trees stretched upwards, like hands begging for help, the fruit have been picked, the songbirds have flew south. This task is the first that requires an elementary analysis of the means of expression of a musical work, but the result of its implementation is: a deeper insight into the emotional world of the piano miniature, elementary but new knowledge about the music means of expression and on what is important to pay attention when trying to reveal the emotional content of a music work. The analysis necessary for the successful solution of the task can be used by the teacher to acquaint the students in more detail with the language of music. This analytical activity is beneficial for the development of students' logical thinking, and the handling of visual-auditory- emotional categories, necessarily, in accordance with the age of the students, contributes to the development of their emotional intelligence.

The next task - to perform an accompaniment to the song "Early Winter", written in children's "score" with "long" (before the musical literacy, which will start in the next school year, the duration "quarter" is denoted by the syllable "ta") and 'short' syllables (the syllable 'ti' is used to indicate duration corresponding to an eighth - example № 3) - is a task for playing with the school musical instruments tambourines and sticks, also marked in the 'score'.

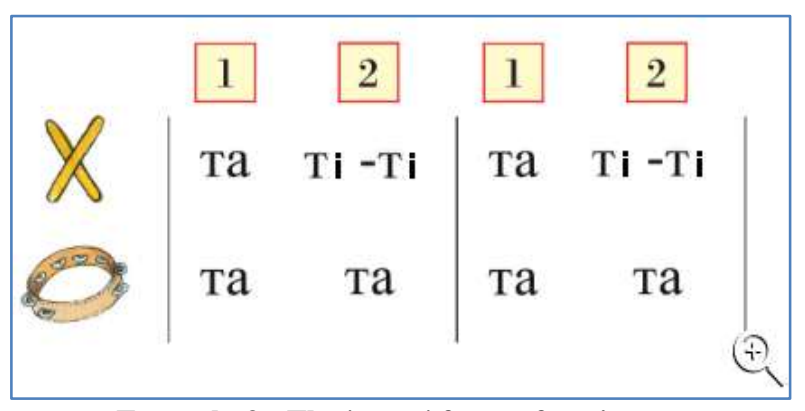

Example-3 - The 'score' for performing an accompaniment to the song "Early Winter"

This task is, given the age of the students, still a basic attempt at reading symbols. Performing accompaniment for singing requires the ability to follow the solo voice /choral performance to achieve synchrony. It is also very necessary to take into account the intensity (volume) of the accompaniment, both not to outvoice the solo part or, conversely, not to be too quiet for it, and the nature of the music to be recreated by the soloists and accompanists. The character of the song is leading for compliance of the student performance. Again, the nature of the song, but this time related to the joy that the first bright rays of the sun and the cheerful noise of the returning migratory birds bring, is decisive in terms of intensity and "temperament" of the accompaniment of the song "Under our roof", which students also accompany by a rhythm depicted on a children's "score".The success of any activity of music performance requires not only insight into the nature of the work that will be performed, but also deeper empathic skills for immersion, "empathy" in the recreated image. When performing an accompaniment, it is necessary, in addition to timing, also emotional synchronization of the music performance between the soloist and the accompanist. In this sense, the tasks of performing accompaniment, even with student percussion instruments, are a step towards enriching the emotional world of the child, developing his emotional intelligence.

Two more electronic resources have been created for the song "Under Our Roof". They are related to the correct response to pitch and metrorhythm. The first resource is the task to determine where the 
beginning of the melody (example № 4) is represented correctly with lines indicating the direction of melodic movement.

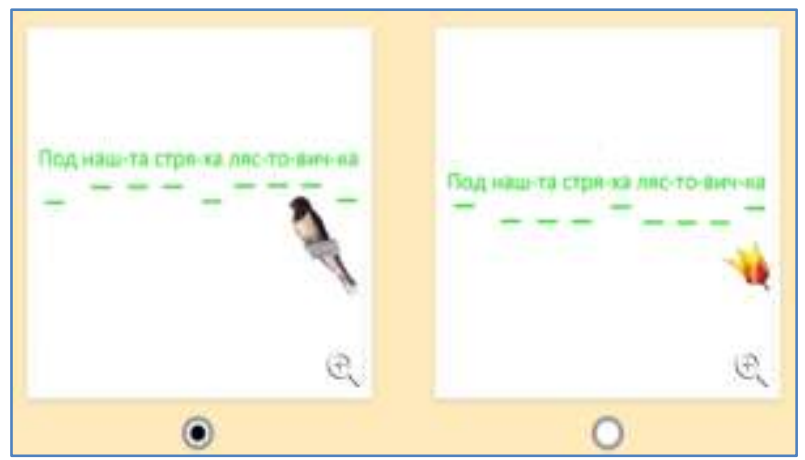

Example-4 - a panel for determining the direction of the melodic movement at the beginning of the song "Under our roof"

For better orientation of the students, the lines depicting sound pitches are placed under the syllables of the poetic text. The panel containing the correct image is illustrated with a swallow (which is the "heroine" of the song), and the board with the wrong answer - with a tulip. Despite the hint of the correct answer, both images are associated with spring and the vitality of this season. In the second task is required to indicate whether the syllables of the poetic text of an excerpt from the song (example № 4a) are correctly marked on a panel on which (since the second-grade students do not yet have music literacy) the rhythm, as in the first year of music education, is marked with the rhythmic syllables "ta" - meaning a medium-long syllable (used instead of a quarter) and "ti" - a short one, used instead of an eighth (example № 4b).

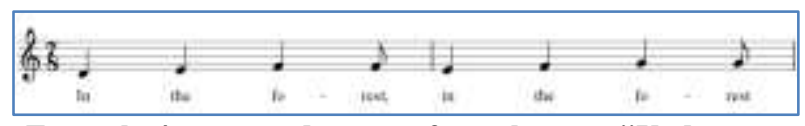

Example-4a - notated excerpt from the song "Under our roof"

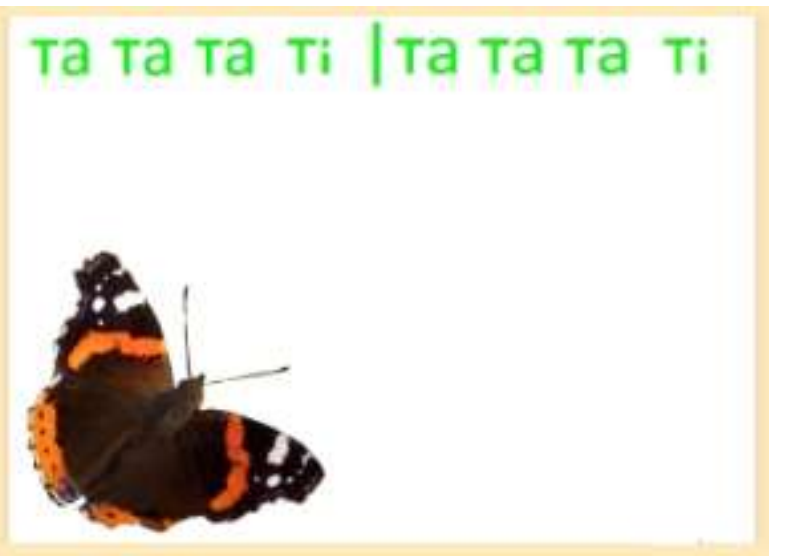

Example-4b - a panel with an excerpt from the song

"Under our roof", on which the rhythm is shown with "rhythmic syllables"
The winter is the coldest season of the year, with the least sunshine. This is the season in which fluffy snowflakes fall slowly from the sky, spinning as if dancing. The tree sap, which usually evaporates, fails to turn into steam on a frosty winter day, but instead wraps itself around the branches, creating beautiful garlands of bristling needles, strings of snow-white popcorn, or, on windy days in high mountains, elongated funnels as if the icy breath of the wind had frozen even the forest fanfares on which he plays. All this, together with the approaching Christmas (25. - 27. 12.) and New Year's (31. 12. - 01.01.) holidays, creates a special atmosphere of silence and purity, of noiseless, excited anticipation. In the printed textbook for the 3rd grade [2, p. 24 - 25] the lesson for the winter is an impressive collection of poems, songs, tasks for creating a rhythmic accompaniment to the listened piano piece "Troika" by P.I. Tchaikovsky, for composing a "sound picture" to a riddle, which in poetic form paints a rich winter picture, for composing a melody on verses and creating an accompaniment to the composed melody. Performing all these tasks, the third-grade children empirically acquire knowledge of the applicability or inapplicability of a particular timbre, dynamic nuance, and melodic intonations to depict specific images and achieve a particular character. To complement all this diversity, three tasks have been added to the electronic version of the textbook. In the condition of the first task it is required to present with geometric figures the structure of "Winter Song" on text by Tsvetan Angelov and music by Parashkev Hadjiev. The song is in a simple two-part form. The proposed groups of figures from which to choose the correct one are: rectangle - circle rectangle (illustrating a simple three-part reprise form), square - circle - triangle (representing a simple threepart form with different first and third parts), two identical ellipses (depicting simple one-part form) and the correct choice: circle - square (corresponding to a simple two-part form). The correct choice would not be difficult for the students, but in this lesson they should abstract from the whole exciting variety of impressions and means with which the winter is presented and choose a certain set of geometric figures that do not correspond to the variety of nuances of dynamics, poetic imagery, metrorhythm, timbres, harmony, but only to the musical structure. The disregard of the actual impressions, no matter how rich and influential they are, and the derivation of the abstract musical form (in this case - the two-part structure verse - refrain), moreover depicted graphically, requires and develops the ability of abstract thinking. In the next task the third-grade children are asked to imagine the delicate falling of the snowflakes and to indicate what character the dance, with which they would recreate their movements in the air, will have, i.e. the figures with which they will recreate the snowflake dance will be:

- Clumsy and slow;

- Temperamental, with a lot of tapping and high jumps;

- $\quad$ Agile and fast; 
- Rough and abrupt;

- Graceful and light. Naturally, for anyone who has seen the snowfall, and in the particular lesson has perceived all the poetic and musical descriptions of the winter, it is easy to point to the last answer as correct. There may be some hesitation with the answer "Agile and fast", which is observed in a snowstorm, but blizzards are not the usual type of snowfall. In this electronic resource a connection is created between the expressive language of music and that of another art - dance. Connections of this type broaden students' aesthetic horizons. Prominent Bulgarian music theorist and pedagogue Professor Penka Mincheva shares: "From the acquired artistic experience (in the perception and exploration of works of other arts) and life experience arise associative conceptual images (always individual), which make the emotional response to listening more complete, more comprehensive, deeper and more lasting " $[6]$.

The third task is to illustrate "Winter Song". In order for the pictures to be successful, in the condition of the electronic resource the students are helped by three questions - what is the mood of the song; what characters the lyrics describe and what colors are appropriate to illustrate the temper in the song and the winter. In this way, not only the character of a tonal work is precisely determined, but this character is also associated with the coloring of a painting, and, as far as depicting the "heroes" described in the verses is demanded - snowflakes dancing carefree, snow-covered pine, whining blizzard, the students should look for appropriate means to portray them, which provokes their imagination and creative thinking.

Spring is the season of the enchanting aromas of the freshly blossomed flowers, bushes and fruit trees, of the cheerful singing of the migratory birds that have just arrived from the warm countries, of the trembling flutter of the impatient to spread their colorful wings graceful butterflies, of the caressing sunbeams more and more often overcoming the clouds and the mists, of both tender and solemn joy of the greening of the plants, "resurrecting" for a new life.

In the printed edition of the textbook for the third grade [2] spring is represented by the song "Spring" on text written by Dimitar Gundov and music by Parashkev Hadjiev, by the piano piece "April. Snowdrop" by P. I. Tchaikovsky, by tasks for determining the character of the two works, by the questions: from what choir can the song "spring" be performed and what dance is appropriate for this song. In the electronic textbook, this is complemented by three tasks. First task is to choose the correct graphic representation of the structure of the song, which is a simple one-part form. The second task requires determining the most appropriate photo corresponding to the character of the song "Spring Fun" with lyrics by
Dimitar Spasov and music by Mihail Shopov. The photographs represent: a tree with leaves painted in golden yellow, behind which a bright blue sky is visible; tulips with a yellow core and red streaks expanding towards the outer edge, with an illuminated inner surface, which gives irradiation to the flowers and with a glare inside the cup of one tulip, but with a dense, heavy shade on the left side of the flowers; sunrise over snow-capped mountain peaks and a sky strewn with soft pink clouds; a sea with a smooth surface and bright sun glints, but the sun, "pushed" into one corner of the photograph, "peeks" over a thick, lead-gray cloud. As can be seen from this description and from the illustration (example № 5), there is an element in each image that gives a certain ambiguity to the temperament. In the autumn photo it is the turquoise blue sky, seen behind the golden leaves; in the second photo - that of the tulips, the dark shade on the left contrasts and "weighs down" on the sunny character of the image; the pink clouds give the winter landscape tenderness, color and caress uncharacteristic for this season; and the thick, dark cloud that occupies most of the sky and to a certain extent obscuring the sun, as well as the menacingly dark color of the seawater in the fourth photograph, did not match our notion of summer. This ambiguity creates some difficulty in working on the electronic resource. The lyrics of the song have no connection with any of the objects depicted in the photos. Then the logic comes to the rescue - the objects associated with the spring season are the tulips - flowers that bloom in spring, as well as the feeling of warmth and mildness caused by the gentle play of sunlight on the smooth surface of the flowers, creating glares like a hinted celebration of the amplifying with each day spring light. This task is similar to the task of finding the most appropriate image to illustrate the song "Autumn". Here, however, the images are richer, not as emotionally unambiguous as those in the first "seasonal" task for the year. This comparison illustrates the gradual complication of the tasks during the educational process.

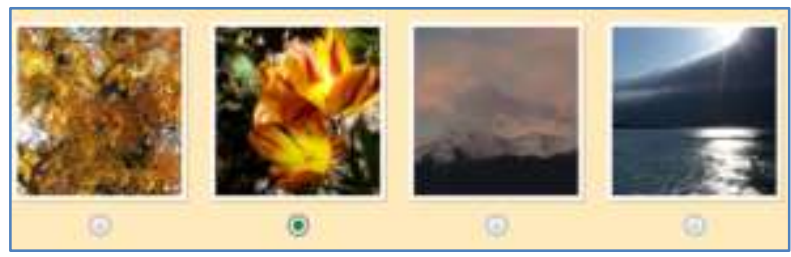

Example-5 Task to select a suitable photo according to the temperament of the song "Spring Fun"

The topic of the seasons in the 4th grade [3] begins with the task of indicating which of the proposed five photos is closest to the nature of the melody and the poetic text of the song "Autumn" with lyrics by Petya Ivanova and music by Plamen Arabov, which the students study and perform in the lesson. The five photos represent one and the same leaves, but each image is modified to express a different emotional nuance (example № 6). The first photograph shows the 
leaves brightly colored in shades of red, clearly visible on a neutral (mostly light gray) background. The second image presents the leaves in a negative - on a very dark gray background the leaves are in dark blue-green shades. In the third photo, the leaves are colored in yellow, earthy brown, but also fresh green. Thus, they represent the diversity of colors of the autumn, but the last element of color to some extent creates an association with spring. In the fourth photo, the leaves are in copper-red tones, but somewhat paler than in the first photo. The last image presents the same leaves, but already in shades of gray, which evoke a distant association with the ice crystals that appear on the windows on a cold and damp winter morning. The lyrics of the song berhyme the fertility, the generosity, the abundance of the "golden autumn", the fact that we will welcome its beauty with joy. In the melody, complemented by rich harmony, there is no trace of autumn despondency, the melodic line is varied, expressive, and the tempo is moderate. Everything in the song suggests the idea of abundance, generosity, wealth, admiration, love. Under these conditions, there may be some hesitation between the three images, which represent autumn colors - photographs in yellowgreen, copper-red and bright red colors. The unadulterated admiration for the generosity and fruitful richness of autumn, which is berhymed, as well as the unequivocal suggestion that the bird songs, the clear blue skies and the carefreeness that characterize spring and summer are over, allude that it is not appropriate to choose neither yellow-green nor the copper-red but somewhat pale leaves, and the picture that most accurately corresponds to the character of the song are the raw bright red leaves of the sumac. By repeatedly solving problems like this - an approach that is applied in the resources of electronic textbooks for the 1 st -4 th grades, gradually develops a fine sensitivity to the shades of the visible colors, the emotional nuances that make up a poetic text and the impact of a music work, as the two types of information that the consciousness deciphers more easily - the visual and the verbal, help to understand the emotional content and the tonal work. Thus, tasks of this type are a step towards the development of students' emotional intelligence.

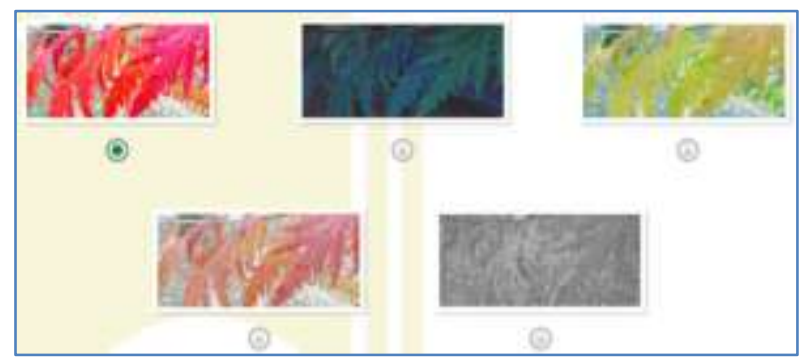

Example-6 Task for selecting an image according to the temperament of a studied song

This electronic resource is followed by a task to illustrate "Four Seasons" by A. Vivaldi, which in its structure and organization is both a series of creative tasks and a collection task. This four-series task is assigned for the fourth grade students in connection with their introduction to the work "Four Seasons" by Antonio Vivaldi. With the onset of each of the seasons, the fourth-graders get acquainted with the part of the respective season provided for listening in the printed textbook. The selected examples from "Four Seasons" are: Part I from "autumn", Part I from "winter", Part I from "spring" and Part III from "summer".

After listening to Part I of "autumn", fourthgrade students are asked to illustrate the music they have listened to. To make it easier for the children, several questions have been prepared, the condition being set that the nature of the drawing corresponds to the nature of the music:

- What will be the coloring - gloomy, cheerful, gentle, subdued, composed of pastel tones, or bright;

- What characters will inhabit the picture - children, animals, fairy creatures, what "costumes" they will wear and what actions they will perform;

- In what environment will the action "unfold" - in a room, city, palace, in nature - at sea, in the mountains, in a cave, in a field, in a forest meadow, or another background will be sought. It is proposed that the finished illustration be saved. With the onset of winter, a lesson is planned, in which fourth-grade students would listen to part I of "Winter" from "Four Seasons" by Vivaldi. After listening to the music, they are again given the same task, this time to illustrate the excerpt from "Winter". The questions that direct the child's imagination are the same, with the aim of creating in the minds of students a continuity between the parts of the musical cycle and, accordingly, between the drawings with which the children illustrate it. Fourth grade students are reminded that each student should keep the illustration of "Winter" with the first one with which he previously painted his "Autumn". The onset of the astronomical spring is marked by a lesson that introduces Part I of "Spring" of the studied musical cycle by Vivaldi. Again, a task with the same elements is offered, now to illustrate "Spring" and is required the newly created drawing to be saved with the first two. In the last lesson before the summer vacation, students listen to Part III of Vivaldi's "Four Seasons" and illustrate it based on the same hints. It is then required the four illustrations to be arranged in a collection that reflects each student's own vision for the season, but also for the music of the Italian composer. In this task there are rich opportunities for comparison - in the preliminary clarifications and comments before illustrating "Winter", "Spring" and "Summer" fourth-grade students are prompted to use the same characters that they drew for "Autumn", but with each subsequent season the question is asked what the characters would be wearing now and what seasonal actions they would 
perform. The "stage sets" can also be compared whether the action takes place in the same environment, or the decors are different for each season, if the backgrounds are different, what requires these decisions. The coloring also provides a favorable basis for comparisons - it very unambiguously recreates the character of the season and the music with which it is depicted. The pedagogue can use all this to create an exciting picture of the change of means of expression needed to recreate a certain emotional content, to recall what musical means of expression are used to depict each season. This is a visually acquired knowledge of the language of music, but at the same time a revision, presented in an exciting way and therefore leaving more lasting traces in students. The drawings themselves can be compared, which gives new opportunities for "explanation" of the expression of the music sounding, of the Vivaldi's palette of means of expression (parts of a large musical work are selected, in which the composer portrays various natural phenomena, i.e. an opportunity is provided to realize the different approaches to depiction with music, but they are all inherent in one author Antonio Vivaldi, who belongs to a stylistic trend Baroque, in the form of Italian Baroque. The introduction of the fourth-grade students to the different parts of this work - a successful idea of the author's team of the educational set, is a definite contribution to building their stylistic culture, but also to establishing the connection between the character of the tonal work and the image created by every student's imagination. The entry of students, not only in the role of artists, but also in those of stage designers, costume designers and, to some extent, screenwriters, as a certain action still has to be depicted in the drawing, greatly enriches their personal contact with various forms of arts.

Another connection of music to the visual arts is presented through a series of two resources in the electronic textbook for the 4th grade. These resources relate to the theme of spring. The goals of this series of resources are again a full insight into the nature of the tonal work, understanding the three-part musical structure, the contrast in the temperament of the second part to the first and third and the similarity of the first and third parts, and acquisition of knowledge of the expressive language of music. The first task requires students to illustrate Edward Grieg's piano piece "To the Spring." In the condition, as well as in the previous electronic resources, questions are proposed, aiding the successful completion of the task, as well as pointing to the connection with the musical structure and the character of the piano piece. The first question refers to the temperament of Grieg's work and whether bright, muted, dark, light, cheerful, sad, "angry" or gentle colors are appropriate for it. The second question points to what characters will be portrayed. The third question is directly related to the structure of the music work, i.e. whether one drawing will be sufficient to show the nature of the music, or whether it is more appropriate to depict it with three drawings (to illustrate the contrast in the mood of the parts). The fourth question directs the attention of the fourth-grade students to the relations of similarity between the first and the third parts, i.e. if the play is being illustrated with three drawings; would the first and the third be similar? The next question concerns the contrasting middle part - whether there is a moment in the musical work that would be represented through a winter landscape. Unlike the first and the third parts, created in major, with a bright idyllic mood and with the higher tones of the middle register mostly used in the melody, the middle part begins with the first motif of the same melody, but now it does not appear in the middle register and in major, as before, but has a changed interval structure, which gives it an anxious sounding in a subdued-low register. This is followed by a sequential transfer of the melody with a third higher, its repeated shortening until a very short structure is reached, "compressed" rhythmically, simultaneously with new transfers in an upward direction and with a gradual increase of tension, after which the culmination "bursts" - bright accented octaves and saturated chords in forte, amplifying to fortissimo. This part is appropriate to be depicted with a drawing, which in its coloring and character contrasts to the first. A winter landscape, such as heavy snowfall, is a good solution. Similar is the sixth question, which is related to the culmination, prepared in the whole middle part and taking place at the end of it. Prompting the nature of the culmination, students are asked if there was a moment in the play that was appropriate to be represented through a storm. By considering the solutions to these questions and, accordingly, the visible images through which they will depict the perceived music, students understand the nature of the contrast of the middle part to the first and the third and the similarity of the both end parts. These relations of similarity and contrast are also the basis for perception and comprehension of the musical form - a simple three-part with small changes in the third part.

The second electronic resource is a video created with the help of $3 \mathrm{D}$ computer animation especially for the music lesson and sounded with the piano piece "To the Spring" by Edward Grieg. This musical work is one of the works for perception in the printed textbook on the topic of spring. In example № 7 a), b) and c) are shown individual frames of the action in the film on the beautiful piano miniature. Because creating a video is a complex and time-consuming task, educational computer films are made to provide as much information as possible about the character of the music, the musical means of expression, the form of the tonal work. The 3D animation provides an opportunity to reflect both smooth and abrupt changes in the character, dynamics, register, and other musical means of expression. Thus, for the beginning of the major, 
bright and tender first and third parts (respectively the first images of each of the series of frames shown in example № 7 a) - for the first part of the piano piece and example № 7 c) - for the third part), which begin with a melody in quiet dynamics and in high register, a colors are selected accordingly in predominantly light tones, with the sparkling whiteness of the snow. On the frames to the left of the examples, which give an idea of the sections of the film synchronous with the first phrase of each part, a snowdrop gradually breaks through the snow, grows and blooms. From the beginning of the second phrase, the snow gradually begins to melt, more snowdrops sprout and bloom. Thus ends the first half-sentence in the music. The next moment - corresponding to the first phrase of the second half-sentence, already presents a delicate spring picture - a tulip gradually unfolding its colorful flower bud, smoothly flowering bush and dandelions. The second and third phrases of the second half-sentence of the musical work are connected. This is presented in the video with the camera no longer being static, but rather creating the illusion of a virtual walk through the area with richly flowering trees and bushes. The third part of the play is not a literal repetition of the first, but has certain changes. In the beginning they refer to:

- The melody, which is now doubled in an octave;

- The accompaniment, which is no longer made up of harmonic chords, but of arpeggios. Since the melody is perceived as the carrier of the main features of the musical "hero", i.e. as the character in the foreground, the appearance of a denser melodic voice (doubling in an octave) in the video miniature is depicted first through the mirror image of the blooming snowdrop that appears in the water, then through the more characters - many snowdrops grow after the first, the tulips in the foreground in the second half-sentence are three, in the second phrase butterflies also appear. This second phrase in the third part of the music work is transposed a quarter higher, which is also reflected in the video impression - while in the first phrase against the background of a sunrise is shown the shore of a lake overgrown with bulrushes, in the second phrase the virtual walk continues in little changed setting - the sun is already higher on the horizon, the flowering trees and shrubs in this part of the shore are more, instead of bulrushes in the lake graceful lilies are blooming, and the butterflies also appear. It is a frame from this moment that is presented in the fourth image of example № $7 \mathrm{c}$ ). The arpeggio accompaniment is recreated through adding a lake for the entire element of the video

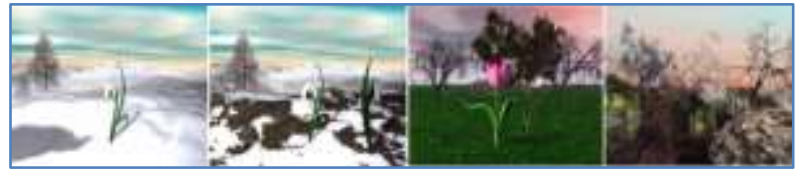

Example-7a - frames from the film, synchronous with the first part from "To the Spring" by E. Grieg story, synchronous to the third part. In the contrasting middle part (example № 7 b) a parallel with a night picture is sought for the low register, and the muffled and anxious sound corresponds to the return of the winter landscape, as well as lighting focused only on the character - the snowdrop, everything else engulfed in dusk. The snowdrop itself loses its petals one by one, shrinks and freezes. In the second part of the ascending sequence, the petals of the tulip wrinkle, fall off and are scattered by a strong wind, and the stem and leaves dry out. In the next part in the sequence, young seedlings bend helplessly under the pressure of the fierce wind. Only one motif remains from the phrase of the music, after its ascending transfer on a third follows its rhythmic "compression", at the same time with which in the film starts snowing, the snow violently quickly covering the ground, and the trees turn white with frost. The culmination is depicted with lightnings flashing synchronously with the accented octaves, as each subsequent flash is brighter, with more branches and illuminates more and more of the mountain over which it falls. Thus, by presenting more and more vividly emotionally colored actions in the film, both the gradual increase of the tension in the musical work and the gradual construction of a culmination, the intensifying of the dynamics, and the deepening of the sounding are revealed. In the fermata, that separates the culmination from the beginning of the third part, the night gradually gives way to the new day, and by mixing the frames a smooth transition to the first frame from the beginning of the third part of the play is achieved, which corresponds to reducing tension and achieving the light, joyful excitement of the music of the last part. This video was made in 2004, but despite the limitations imposed by the capabilities of the software at that time, students continue to perceive it with interest and pleasure. The video is provided for projection before the last task, placed in the printed textbook and, accordingly, visible in the electronic one. It is a task, by using the inspiration that one receives from the spring revival and renewal of nature, each student to create his own song, poem, story, drawing or dance - whatever he wants - and present it to the class. The video contributes to the creative readiness of the fourth-grade students - teachers who worked on the electronic textbook share that after the projection the children are more enthusiastic about creating their own work.

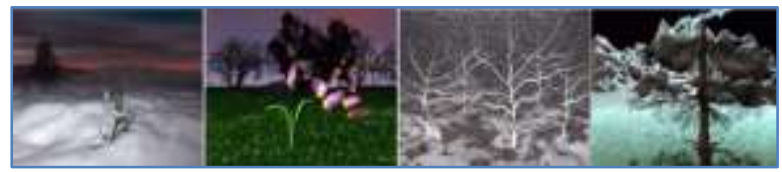

Example-7 b - frames from the film, synchronous with the second part from "To the Spring" by E. Grieg 


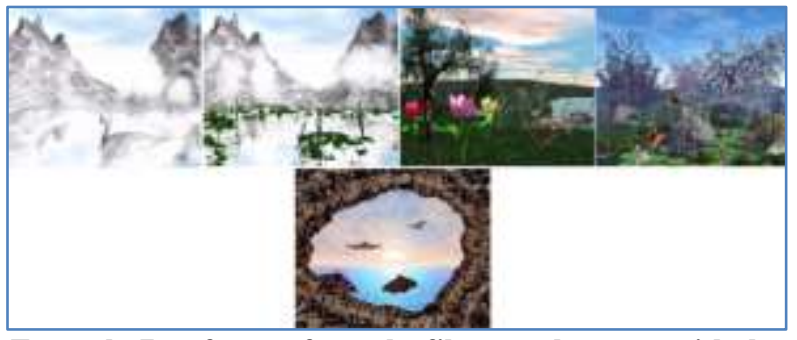

Example-7 c - frames from the film, synchronous with the third part from "To the Spring" by E. Grieg

Experimental verification of the effectiveness of electronic resources related to the topic of the seasons

The pedagogical experiment was conducted in the 2019-2020 school year with students from second, third and fourth grade of general education school divided into experimental and control groups. The final results were taken in the conditions of distance learning.

\section{The tools of the study include}

- Assessment of student success at the initial level (initial ascertaining experiment) and the final level (final ascertaining experiment);

- Test for establishing the creative abilities, made before and after the lessons related to the topic of the seasons;

- Assessment by the pedagogue, who taught the students from the experimental groups, of the interest in the music lessons related to the seasons and the activity shown by the students in the respective lessons.

\section{The participants in the experiment are}

- 41 students from 2nd grade (each of the experimental and control groups consists of two classes), 42 - from 3rd grade, 38 students from 4th grade, forming the experimental classes of a total of 121 participants;

- 42 students from 2nd grade, 40 - from 3rd grade, 41 students from 4 th grade, taking part in the control classes of a total of 123 participants.

The students from the experimental groups use the educational kits, including the electronic textbooks of the author's team led by Prof. Penka Mincheva [1-3]. In the control classes, educational kits of another author's team are used. Since the test tasks intended to solve in the input level (initial ascertaining experiment) and the output level (final ascertaining experiment) are different in the two educational kits, to determine the students' success in the initial and final ascertaining experiment, included in the present study, an equal number of tasks were taken from the two educational kits, and it was explicitly checked that the information needed to solve the selected test tasks was available in the educational kits of both author collectives.
In the initial ascertaining experiment, as well as in the final ascertaining experiment, students were evaluated on two indicators - "success" and "creative thinking".

The success of the students is assessed by teachers, and for registration of their original thinking are used test tasks developed by Asya Veleva [8].

In Bulgaria the evaluation system is six points. This is how the teachers who conducted the experiment assessed the success and creativity potential of students. For the purposes of the research, the success of the students from the experimental and control classes is recalculated, and the grades are equated to:

- Rates "excellent" and "very good" constitute level 1 ;

- $\quad$ Rates "good" - level 2;

- Rates 'medium' and 'weak' - level 3 respectively. Calculated in this way, success is shown in Tables №№ 1 and 2. In order for the results to be comparable by the indicators of success and creative thinking, both when calculating success and when calculating the potential for original thinking, students were initially assessed on a sixpoint scale and subsequently rates are recalculated. After the recalculation of the results by the indicator creative thinking:

- Rates "2" (weak) and "3" (medium) - in the recalculation form "level 3 " and respectively - the prevailing standard thinking;

- Rate "4" is given to those who have shown average success and average propensity for creative decisions, when recalculating they make "level 2";

- Rates "very good" - 5 and "excellent" - 6 form the highest "level 1". These recalculated results are shown in Tables №№ 3 and 4.

Asya Veleva's test tasks [11] were used to study the creative thinking of the students. The modification made (in the present pedagogical experiment the objects with which Veleva worked are not literally borrowed) affects all objects that are used in the tasks. The tasks solved in the present experiment are:

Association [11]. In the initial ascertaining experiment, the students were asked the question "What do you imagine when you hear the word book?" In the final ascertaining experiment, the task is slightly changed, this time an association must be sought with the word box;

- Drawing on a given figure [11]. In the initial ascertaining experiment a wavy line was used, in the final ascertaining experiment - a broken line;

- Drawing different objects from given three identical geometric shapes [11], respectively for the initial ascertaining experiment - three identical squares, and for the final ascertaining experiment - 
three identical rectangles;

- Predicting the effect of a given "impossible" situation [11]. In the initial ascertaining experiment, the question "What will happen if wings sprout on the notebooks?" was used, and in the final ascertaining experiment - the question "What will happen if we walk on springs instead of shoes?"

- Improvement of a specified subject [11]. In the initial ascertaining experiment for this purpose a school backpack was proposed as an object for improvement, and in the final one - a pillow.

- Finding new applications of a subject [11]. Students look for table and cup new applications, respectively.

- Short story about a given character possessing an extraordinary quality [11]. In the initial ascertaining experiment, such a character is a spider as big as a car, and in the final ascertaining experiment - a shoe that can speak.

The diversification of the tasks between the initial and final ascertaining experiment was done for the purposes of the present study, in order to prevent the improvement over time of a response memorized by some students, which would hinder the establishment of correct results.

\section{RESULTS AND DISCUSSION}

According to the indicator creative thinking in the initial ascertaining experiment the results obtained in the experimental and control groups are comparable (Tables №№ 3 and 4. In all tables in which results related to the experiment are reported, the abbreviations IAE - initial ascertaining experiment; and FAE - final ascertaining experiment are used). In the control groups, those who showed medium and low ability to make original decisions are a sufficient number to have the theoretical opportunity to develop and increase their assessment during the training experiment.

The comparable initial results of the students from the experimental and control groups also on the indicator "success" (Tables №№ 1 and 2), as well as the sufficient theoretical probability of ascending development - of increasing the success in the control groups, show that the experiment is correctly staged.

The success in the final ascertaining experiment increased in all learners (Tables №№ 1 and 2 ). This is a testimony for the careful and diligent work of the teachers who conducted the classes, but also to the fact that the attitude of the two teachers to their profession, in turn, is comparable and, in this capacity, contributes to the correctness of the results of the experiment. The higher final success in the experimental groups is explanable. As far as creation is a human need and causes joy in those who practice it, the presence of a large number of creative tasks in the educational kits, which were worked on in the experimental groups [1-3], increases the interest of students in the music lessons (this is evident also from the results of the pedagogical observation carried out in the experimental groups), respectively the success they receive in the final ascertaining experiment.

The success in the experimental groups reported in the final ascertaining experiment is significantly higher than that in the control groups [Tables №№ 1 and 2].

Table-1: Initial and final success of students in age-specific experimental and control groups (grades) - number of students in

\begin{tabular}{|c|c|c|c|c|c|c|c|c|c|c|c|c|}
\hline \multirow{3}{*}{$\begin{array}{l}\text { Assessment } \\
\text { of success } \\
\text { in grades }\end{array}$} & \multicolumn{6}{|c|}{ Experimental groups } & \multicolumn{6}{|c|}{ Control groups } \\
\hline & \multicolumn{2}{|c|}{$\begin{array}{l}\text { 2nd grade - } \\
\text { number of } \\
\text { students }\end{array}$} & \multicolumn{2}{|c|}{$\begin{array}{l}\text { 3rd grade - } \\
\text { number of } \\
\text { students }\end{array}$} & \multicolumn{2}{|c|}{$\begin{array}{l}\text { 4th grade - } \\
\text { number of } \\
\text { students }\end{array}$} & \multicolumn{2}{|c|}{$\begin{array}{l}\text { 2nd grade - } \\
\text { number of } \\
\text { students }\end{array}$} & \multicolumn{2}{|c|}{$\begin{array}{l}\text { 3rd grade - } \\
\text { number of } \\
\text { students }\end{array}$} & \multicolumn{2}{|c|}{$\begin{array}{l}\text { 4th grade - } \\
\text { number of } \\
\text { students }\end{array}$} \\
\hline & IAE & FAE & IAE & FAE & IAE & FAE & IAE & FAE & IAE & FAE & IAE & FAE \\
\hline Level 1 & 20 & 28 & 19 & 26 & 17 & 20 & 23 & 25 & 19 & 20 & 19 & 20 \\
\hline Level 2 & 13 & 9 & 13 & 11 & 12 & 11 & 12 & 10 & 12 & 11 & 11 & 11 \\
\hline Level 3 & 8 & 4 & 10 & 5 & 9 & 7 & 7 & 7 & 9 & 9 & 11 & 10 \\
\hline
\end{tabular}

Table-2: Total initial and final success of the students from the experimental and control groups - number of students in each level

\begin{tabular}{|l|l|l|l|l|}
\hline \multirow{2}{*}{ Assessment of success in groups } & \multicolumn{2}{l|}{ Experimental groups } & \multicolumn{2}{l|}{ Control groups } \\
\cline { 2 - 5 } & IAE & FAE & IAE & FAE \\
\hline Level 1 & 56 & 74 & 61 & 65 \\
\hline Level 2 & 38 & 31 & 35 & 32 \\
\hline Level 3 & 27 & 16 & 27 & 26 \\
\hline
\end{tabular}


Table-3: Initial and final assessments of the creative abilities of students in the age-specific experimental and control groups (grades) - number of students in each level

\begin{tabular}{|c|c|c|c|c|c|c|c|c|c|c|c|c|}
\hline \multirow{3}{*}{$\begin{array}{l}\text { Assessment of } \\
\text { creative } \\
\text { abilities } \\
\text { in grades }\end{array}$} & \multicolumn{6}{|c|}{ Experimental groups } & \multicolumn{6}{|c|}{ Control groups } \\
\hline & \multicolumn{2}{|c|}{$\begin{array}{l}\text { 2nd grade - } \\
\text { number of } \\
\text { students }\end{array}$} & \multicolumn{2}{|c|}{$\begin{array}{l}\text { 3rd grade - } \\
\text { number of } \\
\text { students }\end{array}$} & \multicolumn{2}{|c|}{$\begin{array}{l}\text { 4th grade - } \\
\text { number of } \\
\text { students }\end{array}$} & \multicolumn{2}{|c|}{$\begin{array}{l}\text { 2nd grade - } \\
\text { number of } \\
\text { students }\end{array}$} & \multicolumn{2}{|c|}{$\begin{array}{l}\text { 3rd grade - } \\
\text { number of } \\
\text { students }\end{array}$} & \multicolumn{2}{|c|}{$\begin{array}{l}\text { 4th grade - } \\
\text { number of } \\
\text { students }\end{array}$} \\
\hline & IAE & FAE & IAE & FAE & IAE & FAE & IAE & FAE & IAE & FAE & IAE & FAE \\
\hline Level 1 & 18 & 26 & 17 & 26 & 16 & 19 & 23 & 23 & 18 & 19 & 19 & 21 \\
\hline Level 2 & 15 & 11 & 15 & 12 & 12 & 10 & 12 & 12 & 14 & 13 & 13 & 11 \\
\hline Level 3 & 8 & 4 & 10 & 4 & 10 & 9 & 7 & 7 & 8 & 8 & 9 & 9 \\
\hline
\end{tabular}

Table-4: General initial and final assessments of the creative abilities of the students from the experimental and control groups - number of students in each level

\begin{tabular}{|l|l|l|l|l|}
\hline \multirow{2}{*}{ Assessment of creative abilities in groups } & \multicolumn{2}{|l|}{ Experimental groups } & \multicolumn{2}{l|}{ Control groups } \\
\cline { 2 - 5 } & IAE & FAE & IAE & FAE \\
\hline Ниво 1 & 51 & 71 & 60 & 63 \\
\hline Ниво 2 & 42 & 33 & 39 & 36 \\
\hline Ниво 3 & 28 & 17 & 24 & 24 \\
\hline
\end{tabular}

The differences in the success in music, both in the initial and in the final stage of the experiment are to some extent similar to those in terms of the development of creative thinking. This can be explained by the fact that the three musical activities implemented in general in music lessons - perception, performance and composition of music - contain a creative element. In this respect, however, the two educational kits differ significantly and through this, especially through the presence of a large number of creative tasks both in the printed and the electronic textbooks in the educational kits of Mincheva et al. [1-3], can be explained the much greater upward dynamics in the results of the experimental classes than in the control classes, especially in the indicator "creative thinking".

The educational kits of the two author teams also differ in principle in the focus of their content. In the educational kits of Mincheva et al. [1-3]:

- The most essential, but also relatively small in volume information, which satisfies the requirements of the Ministry of Education and Science, has been determined for obligatory mastering. Additional information for teaching and optional for learning is provided in the teacher's book;

- There are plenty of creative tasks. With such a purpose are created special topics, as is largely the topic of the seasons (which is held successively, lessons to it are rich in impressions, impacting and including a large amount and variety of creative tasks);

- Much space is devoted to beautiful, rich and emotionally colored illustrations.

- In the educational kits of the other author's team:

- The information provided for obligatory mastering is in a much larger amount;

- The tasks for each lesson are mostly related to checking what has been memorized, gathering additional information, practical observations are numerous;

- A theme for the seasons is not provided.

It is especially gratifying that among the students from the experimental groups, who in the initial ascertaining experiment do not show a tendency to creativity and offer solutions to problems with a low degree of originality, in the final ascertaining experiment appear 8 children who begin to give somewhat more original, not, as before, only traditional answers. In this respect, the work with this group needs to be further improved in order to achieve even more satisfactory results. While in the control groups in terms of success less is noted than in the experimental classes, but still there is an upward movement at all levels, in terms of creative solutions the dynamics is weak. In the control classes in the group of those who indicated predominantly traditional approaches and techniques for solution in the initial ascertaining experiment, at the end of the school year there is not a single student who has undergone development in this direction, ie. responding to problems, innovations, challenges has again remained standard. This is even more unsatisfactory given the fact that the success of one student in this group (4th grade student) has increased at the end of the experiment (Table № 1). This shows that in the group there is a relatively active student who has increased his interest in music, and the higher interest in music, which is an art, ie. directly related to creativity, can also mean a higher interest in the beautiful, exciting, subjective, non-standard. In this situation, it is possible that the more interested student will impact the others. This means that the group has a certain potential for development, but it is realized only as the acquisition of more knowledge and not as the development of non-standard thinking.

Significant dynamics is observed in the experimental groups of those showing a medium and high degree of original thinking. In the initial ascertaining experiment, a total of 51 students had the 
highest results in terms of generating non-standard ideas, and those with an average degree of original thinking were 42 , i.e. in the initial results, the groups of medium and high level of creative thinking contain a comparable number of students (since after the primary assessment by the teachers, the groups constituting level 1 and 3, due to the fact that they include two rates, may be somewhat more numerous than the groups with average results - level 2). It is obvious that in the final experiment these results undergo significant changes the group of the most original thinkers has grown to 71 children, and that of those who showed an average result has decreased to 33. As the group of suggesting mostly standard solutions has decreased from 28 to 17 students, ie. 11 children have raised their rate to average, then the "transition" from the average to the high results in terms of creativity is with 20 students. Given that the indicator in the assessment of children now is not the success, ie. not so much the knowledge, but the change in the very way of thinking, in the very ability to study the problem from more than one side, to find a solution that is not always visible at first glance, which does not indicated by everyone, and to find more than one way to solve a problem, it can be argued that the result is very successful. The impact on the ability itself explains the somewhat smaller number - by 3 less - of the participants in the experimental groups who showed high creative potential in the final ascertaining experiment compared to the other indicator - success. The impact on the ability itself explains the somewhat smaller number - by 3 less - of the participants in the experimental groups who showed high creative potential in the final ascertaining experiment compared to the result on the other indicator - success. The distribution of this result by grades (Table № 3) shows that in 4th grade the number of those who increased their ability to make creative decisions is smaller than that of younger students - the total number of those who showed better results at the end of the training is 4 . This is explained by the fact that in 4th grade the first external assessment is forthcoming - in certain disciplines (music is not included) at the end of the school year students across the country are tested, receiving the same tasks, and the solutions are subsequently evaluated by the same independent committee. Concerned about their future results, it is possible that fourth-graders will try harder to "unify" their solutions according to what they expect to be approved by the teachers, respectively by this committee. In the control group of 4th grade, one of the two students who passed from second to first level on the indicator "creative thinking" did not increase his success accordingly. This "disregard" for the established, for the learning of knowledge, the lack of striving for unification, demonstrated in the results of this student, is an indirect confirmation of the interpretation that diligence, learning information, striving to achieve at all costs better success at the expense of unconventional thinking, which fourthgraders are now more afraid to demonstrate and reject it as "unprofitable", has influenced their results (ie. precisely this student who is not interested in his success at any cost, has begun to think even more unconventionally). This is confirmed by the result of the fourth grader from the control group, who has increased his success, moving from level 3 to level 2, but has maintained his initial position on the indicator of creative thinking. As the results show that such concerns are unfounded - in fact, originality has a positive effect on the student's assessment, then in further education children should be led out of the delusion about the low value of the non-standard approach to the tasks.

To a certain extent, the higher number of students in both the experimental and control groups showed very good and excellent success (level 1) compared to their ability to make non-standard decisions (which is observed in both the initial and final ascertaining experiment - tables №№ 1 - 4), can be explained by the diligence, industriousness of these children, but their lack of original thinking. Nevertheless, the relatively large coincidence of the results on the two indicators in all groups speaks in favor of the Bulgarian educational system, where, as evidenced by the results of the study, the original, creative attitude to problems is valued and has a place in forming learners' success.

Although the research was done in the subject of music, and the three musical activities applied in the lessons contain a certain creative element (the largest is in the activity of composing music, relatively smaller in the performance, which in class is usually choral and conducted by the teacher, and the smallest, but still existing - in perception), the tasks through which the presence and degree of development of creative thinking is established in the experiment, are not oriented to the creation, interpretation or perception of works of art (to the creative activities applied in the music lesson as a rule), but in general to the ability to unconventionally approach problems. This means that the musical activities themselves, applied in general in music lessons, develop a certain kind of creative thinking, but not the ability for an original approach to the tasks as a whole, while the study covers more and different varieties. All this gives grounds to be argued that the various tasks that lead in different ways to a creative solution, which abound in the educational kits used by the experimental groups [1,2 and 3], play a crucial role in the better results recorded in these groups in the final ascertaining experiment.

I asked the pedagogue who taught the experimental classes to assess the interest and activity of the students in the lessons, the theme of which is the seasons. Given the age of the children participating in the experiment - 8 - 11 years, they were not asked to self-assess their activity, interest and the usefulness of the lessons united by the theme of the seasons, as it was 
done in various studies with older students. The control classes do not participate in this section of the study, as their textbooks do not include a topic for the seasons. The teacher registered the following results: A total of 89 students showed high activity, medium - 22 and low - 10. This coincides very much with the data on the registered interest, where there are 91 strongly interested, moderate interest shows a total of 23 and weak - 7 (Table № 5).

Table-5: Activity and interest shown in the lessons on the topic of the seasons - number of students in total in the experimental groups

\begin{tabular}{|l|l|l|}
\hline & Activity & Interest \\
\hline High & 89 & 91 \\
\hline Average & 22 & 23 \\
\hline Weak & 10 & 7 \\
\hline
\end{tabular}

It is natural for those who are more active to be more interested in the lessons, which explains the similarity in the results. Some differences can be explained by the fact that, although they are interested and excited about the topic, some children are more shy, which makes them less active. The activity also depends on the type of nervous activity. On the other hand, individual students may be active as an opportunity for personal expression, but the music or the theme of the seasons will excite them less. The registered overall high activity and strong interest unequivocally confirm that the lessons that exploit the theme of the seasons have a definite positive impact on children.

The higher activity and interest in the lessons for the seasons, compared with the success of the students from the final ascertaining experiment and the results registered at the end of the school year by the indicator "creative thinking" suggest an exciting opportunity: since not only the excellent students but also the majority of the students with average achievements are interested in these lessons and take an active part in them, it can be expected that in the following school years, the creatively conducted lessons will have an additional contribution to increase the success of the learners and improve their ability for an original approach to the problems. This will be the subject of future research.

\section{CONCLUSIONS}

- The educational kits, which include a large number and various creative tasks, are beneficial for the success of the students in the subject of music.

- The creatively oriented learning content of the educational kits used in the experimental groups, as well as specifically the theme of the seasons, developed with many creative tasks, contributes to the development of students' creative abilities.

- The theme of the seasons is conducive to the activity and interest of students in music lessons.

\section{REFERENCE}

1. Mincheva, P., Kr. Fileva, Sv. Hristova. (2017). Music for 2nd grade. Sofia, Prosveta.

2. Mincheva, P., Kr. Fileva, D. Katsarova. (2018). Music for 3rd grade. Sofia, Prosveta.

3. Mincheva, P., Kr. Fileva, D. Katsarova. (2019). Music for 4th grade. Sofia, Prosveta.

4. Mincheva, P. (1985). Education of musical hearing through Bulgarian musical folklore. Sofia, Musica

5. Mincheva, P. (1994). Music and intellect. Sofia: Jusautor

6. Mincheva, P. (2017). Perceiving Music-One of Fundamental Activities at Mass School. International Journal of Literature and Arts. Special Issue: Music and Dance, 5(5-1), 2128.

7. Mincheva, P. (2015). Teaching Creative Abilities of Children in Music Education in the General School. International Journal of Literature and Arts. Special Issue: Musical Theory, Psychology, Pedagogy and Performing, 3(5-1), 31-36.

8. Seferinkina, D. (2019). Six benefits of drawing for the development of the young child. Retrieved on 11. 09. 2020. from https://roditel.bg/6-polzi-ot-risuvaneto-zarazvitieto-na-malkoto-dete/

9. Shindarov, I. (2018). Why drawing is so useful for children. Retrieved on 11. 09. 2020 from https://lechenie.bg/zashto-risuvaneto-e-tolkovapolezno-za-detsata

10. Dimitrova, S. (2016). Education in the world and electronic textbooks (1. 4. grade), Varna, Color Print PAK.

11. Veleva, A. (2012). Pedagogy of creativity. Ruse, Printing base at Angel Kanchev Ruse University. 